13

\title{
Кислород на цилиндрическом поликристалле вольфрама
}

\author{
(С) Б.М. Зыков, Т.М. Красненкова, Б.А. Лазба \\ Сухумский физико-технический институт АН Абхазии, \\ Сухум, Абхазия \\ e-mail: kras1946@mail.ru
}

Поступило в Редакцию 1 ноября 2018 г.

В окончательной редакции 20 февраля 2019 г.

Принято к публикации 1 марта 2019 г.

\begin{abstract}
Методами низкоэнергетической $(0-25 \mathrm{eV})$ пороговой электронной спектроскопии и контактной разности потенциалов в вакууме не хуже $2 \cdot 10^{-8}$ Ра изучены пленки кислорода, полученные при $295 \mathrm{~K}, 325-1425 \mathrm{~K}$ (с шагом в $50 \mathrm{~K}$ ) и при $1575 \mathrm{~K}$, в том числе и при последующем прогреве с температуры адсорбции до $1925 \pm 50 \mathrm{~K}$, на цилиндрической поверхности газофазного вольфрамового покрытия толщиной $30 \mu \mathrm{m}$ на сплаве НбЦ-1 $(\mathrm{Nb}-1 \% \mathrm{Zr})$, применяемом в качестве материала коллекторов термоэмиссионных преобразователей тепловой энергии в электрическую в космических ядерных энергетических установках.
\end{abstract}

Ключевые слова: газофазный, поликристалл, вольфрам, цилиндрическая поверхность, термоэмиссионный преобразователь, коллектор, пленки, кислород, адсорбция, прогрев.

DOI: $10.21883 /$ JTF.2019.08.47906.377-18

\section{Введение}

В качестве наиболее эффективного материала коллектора термоэмиссионного преобразователя (ТЭП) все чаще применяется поликристаллическое вольфрамовое $(\mathrm{W})$ покрытие сплава НбЦ-1 ( $\mathrm{Nb}-1 \% \mathrm{Zr})$. Однако принципы получения приемлемых характеристик ТЭП для этого материала неизвестны. Неизвестно, существует ли возможность дальнейшего повышения его эффективности. Поэтому кроме эмиссионно-адсорбционных характеристик необходимо знание химического состояния и для оптимального коллектора, т.е. при одновременных минимальной работе выхода $\varphi_{\min }$ и максимальной термоустойчивости на нм двойной пленочной системы металл-кислород-цезий $(\mathrm{Me}-\mathrm{O}-\mathrm{Cs})$. Они достигаются только при определенном состоянии его окисления [1-8]. Для получения и поддержания в рабочих условиях ТЭП оптимальной пленки $\mathrm{Me}-\mathrm{O}-\mathrm{Cs}$ необходимо знание процессов взаимодействия $\mathrm{O}$ с коллектором. Прямой перенос данных, полученных для монокристаллов [9], не всегда правомерен. Отсюда следует актуальность изучения пленок на поликристаллах фундаментальными методами подобно тому, как это делается для адсорбции на монокристаллах. Поэтому для изучения химического состояния наиболее рациональными представляются специально разработанные нами для этих целей методы [10-17].

\section{Методы исследования}

Фрагмент коллектора с внутренним диаметром $20.4 \mathrm{~mm}$, способы его приготовления и очистки были такими же, как и в [18]. В приборе с 4-х сеточным анализатором энергии $(E)$ электронов тормозящего поля (АТП) [19] применены следующие методы: контактная разность потенциалов в варианте Андерсона (КРП) для определения $\varphi(\Delta \varphi)$, электронная оже-спектроскопия (ЭОС), в том числе и низкоэнергетическая (НЭЭОС) с $E_{p}=220 \mathrm{eV}$, низкоэнергетическая спектроскопия упругорассеянных электронов (НЭСУРЭ) $[16,17]$ для определения энергетической электронной структуры основных и возбужденных состояний электронов в валентной зоне (В3), т.е. химического состояния, и низкоэнергетическая пороговая спектроскопия истинно-вторичных электронов (НЭПСИВЭ) $[16,17]$ для разделения по энергиям химической связи $E_{b}$ аморфных и упорядоченных состояний. Энергия зондирующего (первичного) пучка $E_{p}$ в этих методах низкоэнергетической пороговой электронной спектроскопии (НЭПЭС) непрерывно изменяется от 0 до $25 \mathrm{eV}$. Источник спектрально чистого атомарного кислорода $(\mathrm{O})$ описан в [20]. Давление остаточных газов $P_{\text {res }}$ в рабочих условиях составляет $\leq 2 \cdot 10^{-8} \mathrm{~Pa}, \quad$ а парциальное для $\mathrm{O}-P_{\mathrm{O}}=5 \cdot 10^{-5} \mathrm{~Pa}$ с временем окисления $\sim 20$ min. Температура $T_{\mathrm{O}}$ образца при окислении составляла $295,325-1425 \mathrm{~K}$ (через $50 \mathrm{~K}$ ) и $1575 \mathrm{~K}$, а при прогреве пленок $T_{h}-$ от $T_{\mathrm{O}}$ до $1925 \pm 50 \mathrm{~K}$ с шагом по $T(\Delta T)$ в 25 или $50 \mathrm{~K}$. Перед напуском О на нагретый образец получается спектр УРЭ (СУРЭ) начального состояния, принимаемый за опорный (рис. 1, кривая 2). После окисления при указанном $P_{\mathrm{O}} \mathrm{O}$ откачивается без снижения $T_{\mathrm{O}}$ и после достижении $P_{\text {res }}$ выключается нагрев и регистрируются вначале $\Delta \varphi$, а затем СУРЭ. ПСИВЭ и ЭОС записываются по необходимости, $\Delta \varphi$ отсчитывается от $\Delta \varphi_{0}=0$ для $T=1375 \mathrm{~K} \quad$ в процессе получения начального состояния, т.е. $\Delta \varphi=0$ соответствует максимально возможной концентрации на поверхности остаточного $\mathrm{O}$ после прогрева в сверхвысоком вакууме не хуже $5 \cdot 10^{-8} \mathrm{~Pa}$. При более низких $T$ О поглощается и, как следствие, при этом уменьшается $\Delta \varphi . T_{h}$ ограничивается началом сильного 


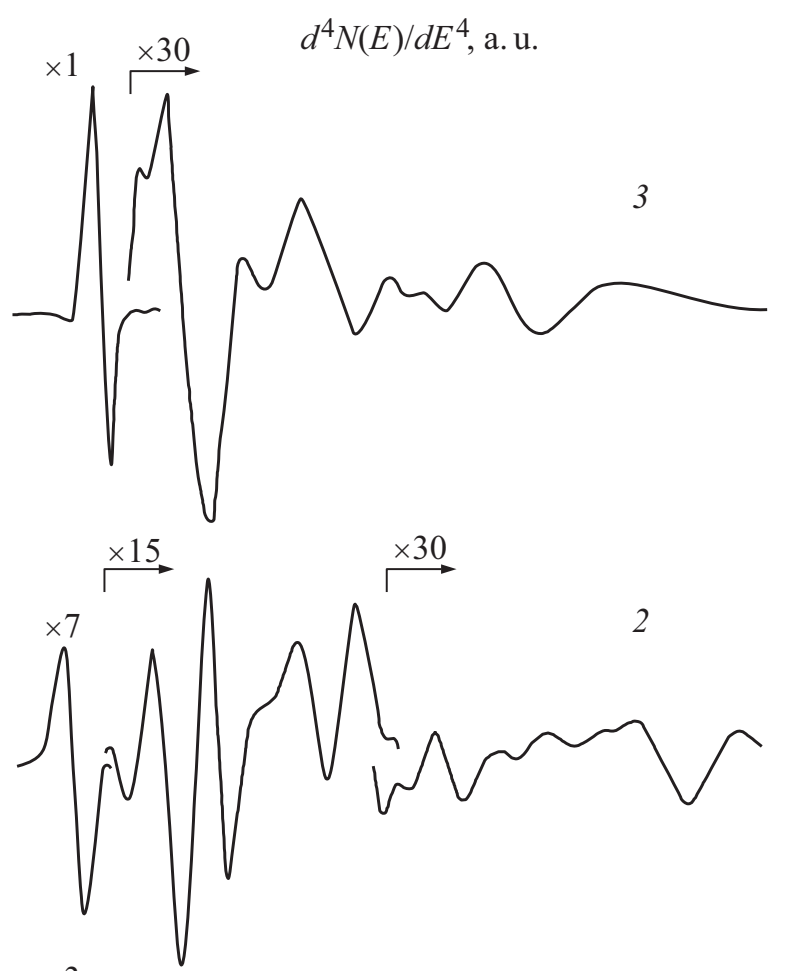

различающихся в области $E=0-2 \mathrm{eV}$. Для одного из них регистрируются два пика - слабой интенсивности $I$ при $0.15-0.25 \mathrm{~V}$ и сильной при 1.05-1.2 V (рис. 1, кривая 1). С других участков записывается только один пик большой I при 0.6-0.7 V (рис. 1, кривая 2). СУРЭ второго типа при остывании образца стабилен. Если образец с СУРЭ первого типа прогревать с постоянным ростом $T$ и не дожидаясь его охлаждения сразу же записывать СУРЭ, то пик при $1.2 \mathrm{~V}$ после $T_{h}=675 \mathrm{~K}$ исчезает, а первый с ростом $I$ и $E$ к $1025 \mathrm{~K}$ достигает $E=0.55 \mathrm{eV}$, т.е. происходит переход к СУРЭ второго типа. Если же не увеличивать напряжение $U$ развертки в СУРЭ выше $3.5 \mathrm{~V}$, то полученный СУРЭ стабилен. Но при повышении $U$ он переходит опять к первому типу, значит, электронный пучок с $E>3.5 \mathrm{eV}$ так же, как и прогрев образца выше $525 \mathrm{~K}$, стимулируют миграцию слабо связанного с подложкой адсорбата. Для О это наблюдается и на монокристаллах $[10-15]$. Но в данном случае возможна еще и миграция СО. Поскольку при этом адсорбат стремится занять на подложке пониженный потенциальный рельеф, из описанного следует, что пик при $1.2 \mathrm{~V}$ в СУРЭ начального состояния отвечает адсорбату в объемных дефектах или на более низких уровнях обычно многоуровневого для поликристалла (в том числе и по энергии) рельефа подложки, а при $0.15-0.7 \mathrm{~V}$ - в поверхностных дефектах или на верхнем уровне, а точнее - поверхностным состояниям $d$-электронов подложки, поляризованным адсорбатом в субмонослойной пленке [10-15]. А именно исчезновение с прогревом второго пика при $1.2 \mathrm{~V}$ и увеличение $I$ и $E$ первого при $0.15-0.5 \mathrm{~V}$ в СУРЭ первого типа означают выход газа из объемных дефектов или с более нижних уровней рельефа на верхний, а обратный процесс миграцию его в обратном направлении. Это же подтверждается и поведением $\varphi$. Измерение $\Delta \varphi$ с точностью до $0.01 \mathrm{eV}$ возможно, в принципе, с $T=1375 \mathrm{~K}$, когда фоновый термоэмиссионный ток с образца становится ниже отсчетного для $\Delta \varphi$ тока $2 \cdot 10^{-8}$ А. После выключения прогрева при $1925 \pm 50 \mathrm{~K} \Delta \varphi$ начинает уменьшаться и в зависимости от числа вспышек при этой $T_{h}$ и $P_{\text {res }} \leq 4 \cdot 10^{-8}$ Ра до достижения $T=500 \pm 20 \mathrm{~K}$ может составлять $-0.25--0.42 \mathrm{eV}$ относительно $\varphi_{0}$. Изменения $\varphi$ обычно резко тормозятся по достижении $\Delta \varphi=-0.25 \mathrm{eV}$. Уменьшение $\varphi$ при охлаждении образца в общем означает переход не десорбировавшегося $\mathrm{O}$, продиффундировавшего при прогреве на верхние уровни рельефа, обратно на нижние или в объемные дефекты, например, по границам зерен. Об аморфности этого адсорбата говорит отсутствие для рассмотренных в СУРЭ пиков энергетических сдвигов $\Delta E$ в ПСИВЭ [10-17]. Необходимость проведения описанного исследования для установления природы указанных пиков в СУРЭ начального состояния вызвана совпадением их $E$ с $E$ пиков в СУРЭ атомночистого W(110), где они стабильны и отвечают собственным поверхностным состояниям [10-15]. Из-за постоянного наличия на поверхности $\mathrm{Nb}$, согласно НЭЭОС, составляющего в приповерхностных слоях около 6.5 at.\% и занимающего не менее 30\% 

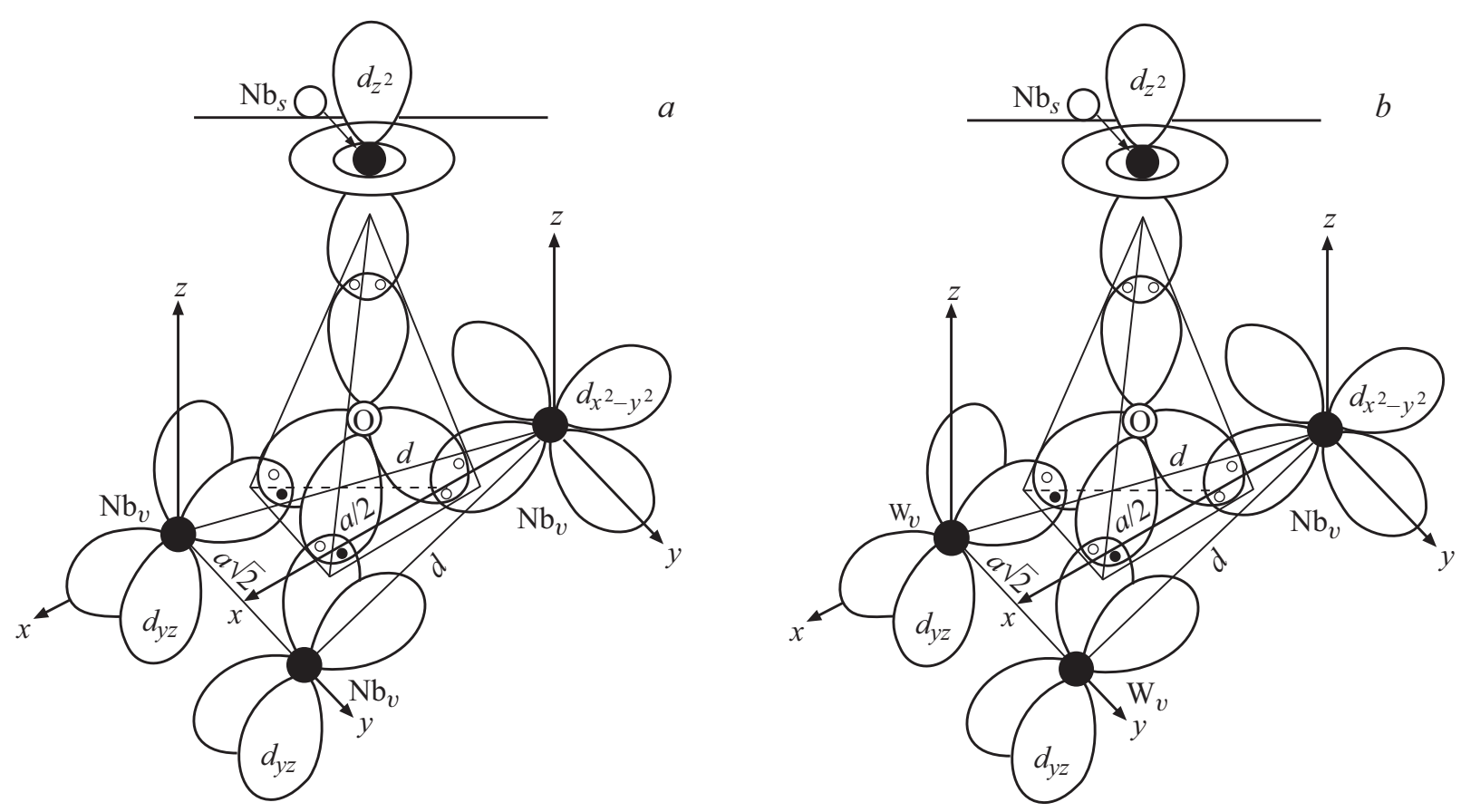

Рис. 2. Схемы валентных связей в субоксидах: $a-\mathrm{Nb}_{4} \mathrm{O}, b-\mathrm{Nb}_{2} \mathrm{~W}_{2} \mathrm{O}$.

ее площади [18], часть О может легко поглощаться и под атомы $\mathrm{Nb}[1-8,10-15]$. С учетом высокой термоустойчивости существующего состояния $\mathrm{Nb}-\mathrm{O}$ можно заключить, что на совершенных участках подложки, скорее всего на нижнем уровне ее рельефа, существует не разрушаемый вплоть до $2075 \mathrm{~K}$ интерметаллический субоксид $\mathrm{Nb}_{2} \mathrm{~W}_{2} \mathrm{O}$ типа $\mathrm{Nb}_{4} \mathrm{O}$ с максимальной насыщенностью связей $\mathrm{Me}-\mathrm{O}[1-8]$ (рис. 2, $a, b$ ). Наличие под верхним слоем атомов подложки О в химической связи само по себе способно приводить к понижению $\varphi$ поверхности [1-8]. При перемещении пучка по поверхности образца удается, как и для W(110) [1-8], найти после высокотемпературного прогрева участки без адсорбата. При этом в СУРЭ второго типа перед пиком при $0.5 \mathrm{~V}$ записывается автоионизационный минимум вблизи нуля (при $0.05 \mathrm{~V}$ ) [10-15]. Для начальных состояний переходного типа, когда с некоторых участков газ уже перешел на нижние уровни и в объемные дефекты и поэтому в СУРЭ отсутствует первый пик, с плавным переходом от уровня фона записывается широкий минимум при $0.6 \mathrm{~V}$, ни при каких условиях не наблюдаемый для монокристаллов [10-15]. Исходя из слабого наклона его левой ветви и отсутствия пиков при меньших $E$ это означает отсутствие для очищенных участков подложки дискретных максимумов плотностей собственных поверхностных состояний $d$-электронов, т. е. существование их набора по всей ширине В3, как, например, для внешних $s$-электронов. Тогда с учетом различной вероятности возбуждения (внутризонных переходов) этих электронов с различными энергиями химических связей $E_{b}$, зависящими от типа дефектов, характера рельефа и различной концентрации каждого из этих типов, левую ветвь описываемой кривой можно представить в виде огибающей множества пиков с близкими $E$. Эти пики можно было бы выявить применяя более высокий порядок электрического дифференцирования (например, шестой). В методах НЭПЭС (т.е. СУРЭ и ПСИВЭ) распределению электронов в энергетических уровнях кристаллического твердого тела как сильносвязанной квантовой системы соответствуют недифференцированный спектр и любая из его четных производных $[16,17]$. Поскольку распределение электронов в этих уровнях относительно максимумов плотности их состояний в обе стороны от них (максимумов) экспоненциально, многократное дифференцирование этого распределения не приводит к появлению ложных пиков. Это отличает такие распределения от распределения электронов вблизи нагретого катода, где они описываются гауссовой кривой, имеющей точки перегиба, вызывающие в спектре при многократном дифференцировании появление ложных пиков. Но, к сожалению, при увеличении порядка дифференцирования уменьшается отношение сигнал/шум, соответственно приводя к уменьшению чувствительности методов СУРЭ и ПСИВЭ $[16,17]$. Естественно, что наибольший вклад в наблюдаемый эффект вносят чистые основания спиральных террас винтовых дислокаций [21], но с адсорбатом в желобах. Это явление аналогично поляризации атомов гладкой подложки адсорбатом в субмонослойной пленке. Пики же при $\sim 4$, $\sim 6$ и $\sim 9 \mathrm{~V}$, не исчезающие ни при каких условиях, явно отвечают сильным химическим связям с подложкой при очистке $\mathrm{O}$ и $\mathrm{C}$, и не отвечают рассмотренному выше состоянию адсорбата. $\Delta E$ пика при $3.7 \mathrm{~V}$ составляет в ПСИВЭ $0.6 \mathrm{eV}$, при 5.6-0.3 eV, а при 8.8-0.1 eV. Значит, субоксид $\mathrm{Nb}_{2} \mathrm{~W}_{2} \mathrm{O}$, которому отвечают два первых пика, упорядочен, т.е. находится на в меру совершенных и 
гладких участках подложки [10-15], а С (в общем случае в виде карбида $\left.\mathrm{W}_{2} \mathrm{C}\right)$ с $E_{b}=8.8 \mathrm{eV}$ для трехцентровой (мостиковой) связи $\mathrm{W}-\mathrm{C}-\mathrm{W}-$ в основном в объемных дефектах, но так как $\Delta E \neq 0$, в слегка протяженных, например, по границам зерен. Исходя из того, что $\Delta E$ для пика при $5.6 \mathrm{~V}$ меньше, чем при $3.7 \mathrm{~V}$, хотя оба они отвечают связям $\mathrm{O}-\mathrm{Me}$ в $\mathrm{Nb}_{2} \mathrm{~W}_{2} \mathrm{O}$, первая - объемным $\mathrm{W}-\mathrm{O}-\mathrm{W}$ и $\mathrm{Nb}-\mathrm{O}$, а вторая - поверхностной $\mathrm{Nb}-\mathrm{O}$ (рис. $2, b$ ), можно заключить, что в других неупорядоченных объемных дефектах имеется $\mathrm{O}$ в мостиковой связи $\mathrm{W}-\mathrm{O}-\mathrm{W}$, вносящий вклад в пик объемных связей от $\mathrm{Nb}_{2} \mathrm{~W}_{2} \mathrm{O}$, а неупорядоченность О уменьшает суммарный сдвиг этого пика. Очевидно, что без применения НЭПСИВЭ разделение таких состояний $\mathrm{O}$ с помощью одной лишь НЭСУРЭ невозможно [10-15]. Пик в СУРЭ при $2.2-2.8 \mathrm{~V}$ (со средней $E_{m}=2.5 \mathrm{eV}$ ) отвечает на монокристаллах $E_{b} \mathrm{O}-\mathrm{Me}$ в неупорядоченных поверхностных дефектах [1-8,10-15] в основном в виде отдельных молекул типа $\mathrm{Me}_{2} \mathrm{O}$. Но в данном случае $E_{b}$ для О изза плохого для поликристаллической подложки дальнего порядка в пленках, очевидно, уменьшены в сравнении с монокристаллической. Кроме того, на данной подложке присутствует еще и СО. Идентичность же внешних несвязывающих орбиталей $\mathrm{O}$ в молекулах $\mathrm{W}_{2} \mathrm{CO}$ и $\mathrm{W}_{2} \mathrm{O}$ должна приводить к почти равным значениям $E_{b}$ для пиков мостиковых связей $\mathrm{W}-\mathrm{C}-\mathrm{W}$ и $\mathrm{W}-\mathrm{O}-\mathrm{W}$. Правда, при наличии на подложке плотноупакованных (ПУ) рядов атомов, имеющих перпендикулярные ей вакантные

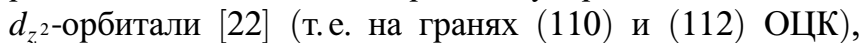
за счет линейной донорно-акцепторной связи $\mathrm{W}-\mathrm{C}$ возможно образование дипольных молекул субкарбонила WCO в основном состоянии, т.е. с тройной связью между С и О (одна из которых сильная линейная) и разнонаправленными заполненными орбиталями С и О [23]. Но на поликристалле такой тип субкарбонила, видимо, не является преобладающим, и его наличие не должно сильно влиять на интегральные химические свойства пленок. Поэтому здесь и рассматривается более общий случай образования $\mathrm{W}_{2} \mathrm{CO}$ с возбужденной, но зато более естественной для атома С в соединениях, тетраэдрической $s p^{3}$-конфигурацией валентных орбиталей. Ясно, что в обоих случаях молекулы WCO или $\mathrm{W}_{2} \mathrm{CO}$ должны располагаться на подложке вертикально, т. е. СО для связи с W должен выбирать на ней гладкие участки, параллельные базовой (усредненной) поверхности. Двойной связи $\mathrm{C}-\mathrm{O}$ в $\mathrm{W}_{2} \mathrm{CO}$ и соответствует пик при $\sim 7.5 \mathrm{~V}$ в СУРЭ начального состояния. При адсорбции О его $E$ может изменяться от 5.9 до $7.8 \mathrm{eV}$. При этом рост его $I$ прямо говорит о том, что он не отвечает плазмонам [10-15]. А после прогрева при 1325 - $1375 \mathrm{~K}$ он вообще исчезает из СУРЭ. Одновременно из ЭОС исчезают пики от СО и карбида [18]. Несмотря на трудности в идентификации на данном этапе пика С с $E_{m}=2.5 \mathrm{eV}$ можно указать, что значительная часть адсорбата с этой $E$, согласно ПСИВЭ $(\Delta E=0.65 \mathrm{eV})$, упорядочена. Соответствие этой пленки $\mathrm{W}_{2} \mathrm{CO}$, согласно указанным особенностям в расположении на подложке таких молекул, не вызывает сомнений. Однако в случае расположения молекул $\mathrm{W}_{2} \mathrm{O}$ в желобах террас, в том числе и винтовых, или бороздках от выхода на поверхность линейных дислокаций и у этой пленки появляются одномерная упорядоченность и отвечающий ей сдвиг пика в ПСИВЭ, хотя ясно, что сдвиг и должен быть меньше наблюдаемого. Таким образом, остается считать пик с $E_{m} \sim 2.5 \mathrm{eV}$ суммарным от $\mathrm{W}_{2} \mathrm{CO}$ и $\mathrm{W}_{2} \mathrm{O}$ до тех пор, пока не выяснится вклад каждого из них. Пик дифракции медленных электронов (ДМЭ) от подложки, для $\mathrm{W}(110)$ наблюдаемый при $13.6-13.9 \mathrm{~V}$, а в этой работе при 13.1-13.5 V, для поликристалла, естественно, обладает меньшей $I$, чем для $\mathrm{W}(110)$ [10-15] (рис. 1, кривые 1,2$)$. Исходя из условий Вульфа-Брэгга пик при $17.3 \mathrm{~V}$ можно считать дифракционным второго порядка. Природа же пиков при 12.3 и $15.3 \mathrm{~V}$ становится ясной только при исследовании адсорбции О. Пики, отвечающие поверхностным и объемным $s$ - и $d$-плазмонам $(E$ в интервалах 5-11 и 17-22 eV), здесь не рассматриваются, так как для поликристаллической поверхности они не вносят большого вклада в $I$ пиков другой природы исходя из того, что их $I$ сильно зависит от степени упорядоченности структур подложки и пленок.

\section{Адсорбция кислорода}

В начальной стадии адсорбции кислорода при $295 \mathrm{~K}$ в СУРЭ резко падает $I$ первого пика при $0.7 \mathrm{~V}$, так что при $\Delta \varphi>-0.1 \mathrm{eV}$ он исчезает. При $\Delta \varphi=-0.19 \mathrm{eV}$ исчезает пик при $2.2 \mathrm{~V}$. Затем при $\Delta \varphi=0.35 \mathrm{eV}$ появляется слабый пик при $1.05 \mathrm{eV}$, но при $\Delta \varphi=0.64 \mathrm{eV}$ и он исчезает. $\Delta \varphi_{\max }$ составляет $0.82 \mathrm{eV}$, т.е. с учетом начального отрицательного $\Delta \varphi$ изменение $\varphi$ происходит на $1.1-1.25 \mathrm{eV}$ и появляется слабый пик при $1.05 \mathrm{~V}$. СУРЭ от состояния с $\Delta \varphi_{\max }$ приведен на рис. 1 , кривая 3. I пиков при 3.7 и $5.7 \mathrm{~V}$ от $\mathrm{Nb}_{2} \mathrm{~W}_{2} \mathrm{O}$ при адсорбции $\mathrm{O}$ падает, причем последний при $\Delta \varphi=0.75 \mathrm{eV}$ исчезает. Пик при $10.75 \mathrm{~V}$ от $\mathrm{O}$ на $\mathrm{Nb}_{2} \mathrm{~W}_{2} \mathrm{O}$ тоже уменьшает $I$, но исчезает при $\Delta \varphi=0.64 \mathrm{eV}$. Падает $I$ пика ДМЭ при $13.25 \mathrm{~V}$, но он из СУРЭ не исчезает. При $\Delta \varphi=0.50 \mathrm{eV}$ в СУРЭ начинаются более резкие изменения. Одновременно появляются минимум при $1.25 \mathrm{~V}$ и два пика - при 1.45 и $4.0 \mathrm{~V}$. Хотя до получения $\Delta \varphi_{\max }$ остается всего $0.3 \mathrm{eV}$, тем не менее их $I$ начинает сильно возрастать, превышая при $\Delta \varphi_{\max } I_{0}$ для минимума при $1.25 \mathrm{~V}$ в 5 раз, а для пика при $1.45 \mathrm{~V}$, к окончанию адсорбции смещающегося к $1.85 \mathrm{~V}$, в 30 раз. I же минимума с $E_{0}=1.4 \mathrm{eV}$, который теперь становится вторым, растет в 8 раз, а его $E$ достигает $2.45 \mathrm{eV}$. Пик при $4.0 \mathrm{~V}$, при $\varphi_{\max }$ достигающий $E=4.3 \mathrm{eV}$, успевает превысить $I$ оставшегося в СУРЭ пика при $3.5 \mathrm{~V}$ в $\sim 1.5$ раза. При $\Delta \varphi=0.35 \mathrm{eV}$ начинается одновременный рост $I$ еще трех пиков - при $7.3,8.85$ и $12.5 \mathrm{~V}$. Их $I$ растет до получения $\Delta \varphi_{\max }$, но увеличившись для двух первых соответственно в 3 и 1.5 раза к $\Delta \varphi=0.64 \mathrm{eV}$ и для третьего в 3 раза к $\Delta \varphi=0.50 \mathrm{eV}$, затем резко падает, достигая к $\Delta \varphi=0.75 \mathrm{eV}$ для первого постоянной величины, такой, как при $\Delta \varphi=0.10 \mathrm{eV}$, а для второго - 
$I_{0}$. При дальнейшей адсорбции $\mathrm{O}$, хотя до получения $\Delta \varphi_{\max }$ остается всего $0.07 \mathrm{eV}, I$ второго пика падает еще в 1.7 раза, а третьего - до ступеньки. С начала адсорбции О постоянно растет $I$ лишь одного пика при $15.4 \mathrm{~V}$, увеличиваясь при $\Delta \varphi_{\max }$ в 3.5 раза. В ПСИВЭ для $\Delta \varphi_{\max }$ пики СУРЭ при $1.85,4.3,6.95,8.85$ и $15.15 \mathrm{~V}$ имеют $\Delta E=0.3 \mathrm{eV}$, при $11.7-0.55 \mathrm{eV}$, при $13.1-0.15 \mathrm{eV}$, а для $3.55,18.1$ и $20.05 \mathrm{eV} \Delta E=0$. Большая величина $\Delta E$ ( $\sim 0.6 \mathrm{eV}$ и выше) указывает на преобладание совершенной пленки, малая $(<0.3 \mathrm{eV})-$ на преобладание в ней малых островков, а $\Delta E=0$ отвечает аморфной [10-15]. Согласно приведенным данным, процесс адсорбции О можно представить следующим образом. Вначале экранируются участки, не полностью занятые слабосвязанным $\mathrm{O}$ с $E_{b}=2.2 \mathrm{eV}$, причем поверхностные дефекты раньше объемных, так как вначале исчезают пики при 0.7 и $2.2 \mathrm{~V}$ и только затем появляется и исчезает пик при $1.05 \mathrm{~V}$. Одновременное падение $I$ пиков ДМЭ при 13.25 и $17.3 \mathrm{~V}$ означает, что слабосвязанный адсорбат с $E_{b}=2.2 \mathrm{eV}$ может располагаться и на гладких участках подложки, например, ступеньках или террасах ниже уровня наиболее выступающих над базовой поверхностью вершин из материала подложки. Одновременно экранируются участки с наиболее устойчивым $\mathrm{Nb}_{2} \mathrm{~W}_{2} \mathrm{O}$, причем его объемные связи с $E_{b}=5.7 \mathrm{eV}$, естественно, сильнее поверхностной с $E_{b}=3.7 \mathrm{eV}$. Адсорбция $\mathrm{O}$ на нижнем уровне рельефа и в дефектах в отличие от совершенных участков на более верхних может происходить и многослойно. Об этом говорит экранирование уже с самого начала адсорбции несвязывающих орбиталей $\mathrm{O}$ с $E=10.75 \mathrm{eV}$ на нижнем уровне между молекулами $\mathrm{Nb}_{2} \mathrm{~W}_{2} \mathrm{O}$. На монокристаллах же при адсорбции $\mathrm{O}$ этот пик только возрастает, так как там не может быть более чем двухслойной адсорбции. Причем там он еще и подчиняется условиям дифракции [10-15], т.е. точно отвечает упорядоченно адсорбированному О на субоксиде. В последней стадии покрывается верхний уровень рельефа, т. е. плоскости самых верхних ступенек или террас, выступающих над участками, занятыми начальными соединениями и к этому моменту уже экранированными О. При этом оказывается возможным образование на них небольших островков субоксида, скорее всего $\mathrm{W}_{3} \mathrm{O}$, который из-за повышенного при этом $P_{\mathrm{O}}=5 \cdot 10^{-5} \mathrm{~Pa}$ (иначе невозможно получение $\Delta \varphi_{\max }$ ) почти сразу же начинает экранироваться. Отвечающий ему пик при $4.0 \mathrm{~V}$ по достижении $\Delta \varphi_{\max }$ увеличивает $E$ до $4.3 \mathrm{eV}$. Такой рост $E$ прямо отвечает накоплению адсорбата в слабо упорядоченном состоянии, так как в аморфном растет только I. Появление в конце адсорбции пика при такой сравнительно большой для данной системы $E$ не удается объяснить никаким другим механизмом. При образовании островков $\mathrm{W}_{3} \mathrm{O}$ одновременно происходит и сильное экранирование $\mathrm{CO}$ и $\mathrm{W}_{2} \mathrm{C}$. Кроме того, рост $E$ пика при $1.45 \mathrm{~V}$ до $1.8 \mathrm{~V}$ сам по себе и также ее значение говорят о накоплении О в протяженных дефектах уровней, по крайней мере, не выше среднего, например, хотя бы в желобах террас. Особенности в поведении $\mathrm{CO}$ и $\mathrm{W}_{2} \mathrm{C}$ при адсорбции $\mathrm{O}$ объясняются следующим образом. О, который на поверхности может образовывать сильную двойную (или тройную [23] при наличии на подложке ПУ рядов ее атомов) связь с C, разрывает в объемном дефекте менее сильную мостиковую связь $\mathrm{W}-\mathrm{C}-\mathrm{W}$ в молекулах аморфного $\mathrm{W}_{2} \mathrm{C}$ и в стремлении образовать сплошную пленку в химической связи с подложкой переносит С в составе $\mathrm{CO}$ на более верхние гладкие участки, где имеется больше возможностей для образования оптимальной пространственной ориентации внешних орбиталей атомов подложки и соседних молекул. Образующиеся при этом по описанному выше механизму молекулы $\mathrm{W}_{2} \mathrm{CO}$ (или WCO) из-за необходимости вертикального расположения могут накапливаться только на гладких участках, параллельных базовой поверхности, например, на основаниях террас, и образовывать в меру упорядоченную пленку, что понижает $E$ системы. Молекулы же $\mathrm{W}_{2} \mathrm{C}$ могут образовываться и на отчасти наклонных стенках или вблизи них. Но из-за наличия дефектов даже на гладких участках часть вновь образовавшегося СО не сможет образовать молекул $\mathrm{W}_{2} \mathrm{CO}$ и поэтому будет вынуждена отдавать О и снова выделяться (но теперь уже на более верхних уровнях) в виде $\mathrm{C}$, тут же образующего карбид $\mathrm{W}_{2} \mathrm{C}$ с материалом подложки. Поэтому при адсорбции $\mathrm{O}$ и наблюдается одновременный рост в СУРЭ пиков при 7.3 и $8.8 \mathrm{~V}$. Этот эффект происходит прежде всего там, где объемный дефект граничит с узкими террасами (карнизами) на наклонных стенках „Пиков““ или „хребтов“, вершины которых расположены выше самых верхних плоских ступенек. Т. е. стенки объемных дефектов, как и винтовые дислокации $[9,16,17]$, могут содержать многоуровневые террасы. Ясно, что только для такого рельефа при дальнейшей адсорбции О возможно экранирование карбида и вертикально расположенных молекул $\mathrm{W}_{2} \mathrm{CO}$ или WCO. C другой стороны, если наблюдается экранирование СО, то, следовательно, он не может присутствовать на самом верхнем уровне рельефа, где в конце адсорбции $\mathrm{O}$ образуется $\mathrm{W}_{3} \mathrm{O}$.

\section{Прогрев пленки кислорода, полученной при $295 \mathrm{~K}$}

Рассмотрим процессы, происходящие на поверхности $\mathrm{W}$ покрытия при прогреве пленки О с $\Delta \varphi_{\max }$, полученной при $295 \mathrm{~K}$. При прогреве начиная с $295 \mathrm{~K}$ в СУРЭ (рис. 1) происходит падение $I$ пика при $1.85 \mathrm{~V}$, сопутствующих ему минимумов при 1.25 и $2.45 \mathrm{~V}$, пиков при $3.5,4.3,13.1$ и $15.5 \mathrm{~V}$. После $600 \mathrm{~K}$ минимум при $1.25 \mathrm{~V}$, самый интенсивный пик при $1.85 \mathrm{~V}$ и пик при $3.5 \mathrm{~V}$ исчезают. При этом минимум при $2.45 \mathrm{~V}$ смещается к $1.85 \mathrm{~V}$. Пик при $4.3 \mathrm{~V}$ уменьшает $E$ до $4.0 \mathrm{eV}$. Пик же при $13.1 \mathrm{~V}$ исчезает уже при $425 \mathrm{~K}$. При $575 \mathrm{~K}$ появляется пик при $5.65 \mathrm{~V}$ от $\mathrm{Nb}_{2} \mathrm{~W}_{2} \mathrm{O}$, при $625 \mathrm{~K}$ появляются пик при $0.8 \mathrm{~V}$ от поляризованных состояний электронов подложки и новый при $3.3 \mathrm{~V}$ от О на протяженных терраcax [1-8]. Затем при $675 \mathrm{~K}$ появляется пик при $2.7 \mathrm{~V}$ от адсорбата на поверхностных дефектах среднего уровня 


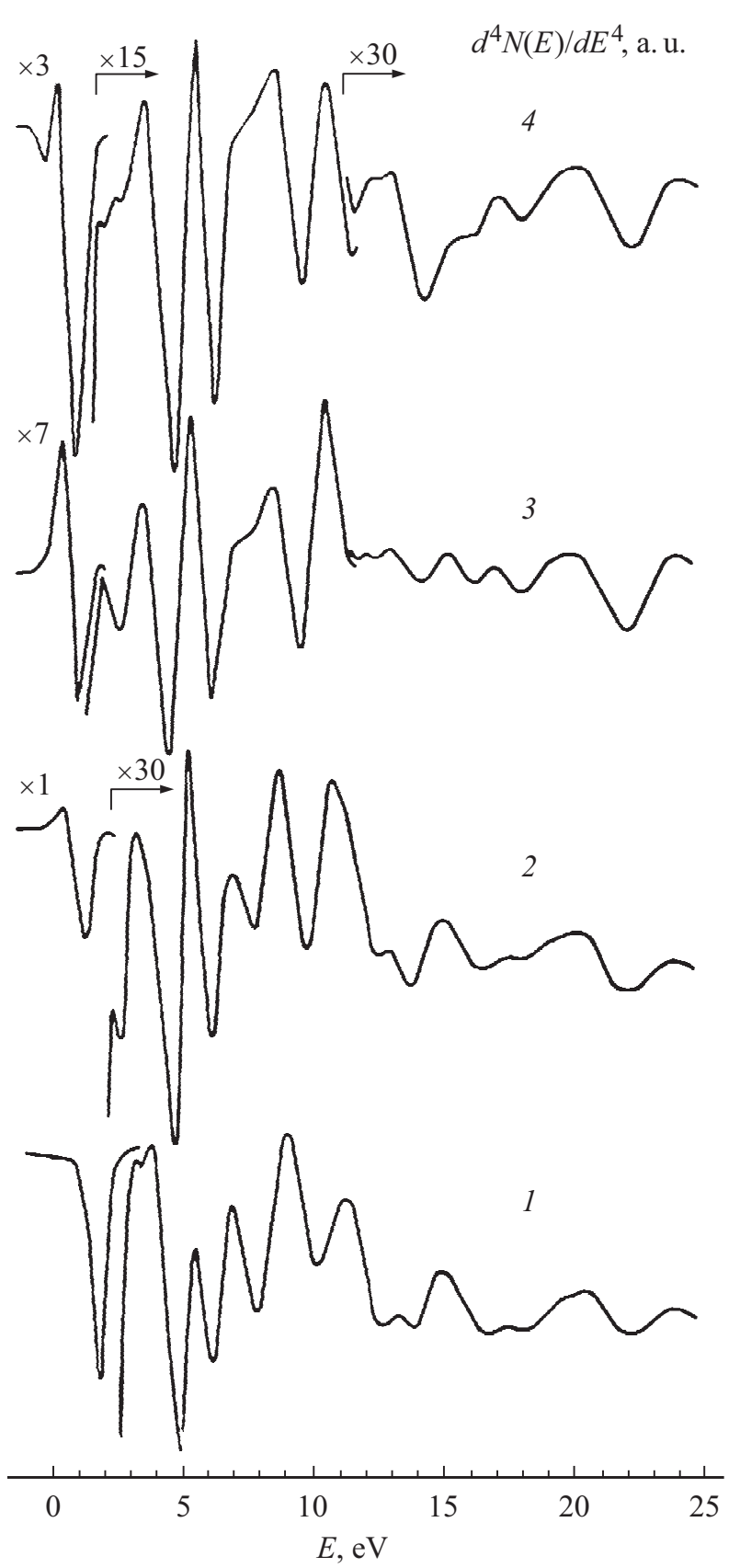

Рис. 3. Спектры УРЭ при прогреве пленки с $\Delta \varphi_{\max }$, полученной при $T_{\mathrm{O}}=295 \mathrm{~K} .1-$ десорбция $\mathrm{O}$ с верхних уровней рельефа подложки $\left(T_{h}=475 \mathrm{~K}\right) ; 2-\mathrm{O}$ на среднем и нижнем уровнях рельефа подложки $\left(T_{h}=700 \mathrm{~K}\right) ; 3$ - десорбция $\mathrm{CO}$ $\left(T_{h}=1325 \mathrm{~K}\right) ; 4$ - участки подложки, отличные от тех, которым соответствуют спектры на рис. 1 (кривая 1 и 2 после прогрева при $T=2025 \mathrm{~K}$ ).

рельефа. При $700 \mathrm{~K}$ новый пик при $3.3 \mathrm{~V}$, увеличив $E$ до $3.65 \mathrm{eV}$, сливается с пиком при $4.3 \mathrm{~V}$, который, в свою очередь, уменьшает $E$ до этой величины. Значит, начиная с этой температуры прогрева $T_{h}$ пик при $3.65 \mathrm{~V}$ уже отвечает $\mathrm{Nb}_{2} \mathrm{~W}_{2} \mathrm{O}$ (рис. 3, кривая 2). С начала прогрева и до $625 \mathrm{~K}$ растут интенсивность $I$ и $E$ пика при $7.0 \mathrm{~V}$ от СО. С температуры $650 \mathrm{~K}$ его $I$ начинает падать, что отвечает частичной десорбции СО. Поэтому учитывая, что $T$ полной десорбции СО $(1325-1375 \mathrm{~K})$ значительно выше этой $T_{h}$ (рис. 3, кривая 3), а также учитывая необходимость вертикального расположения молекул $\mathrm{CO}$, можно заключить, что адсорбция $\mathrm{CO}$, скорее всего, многоуровневая. При $550 \mathrm{~K}$ начинается рост $I$ пика при $8.85 \mathrm{~V}$ от $\mathrm{W}_{2} \mathrm{C}$, достигая при $625 \mathrm{~K}$ увеличения в 2 раза относительно его $I$ при $295 \mathrm{~K}$. Но выше $650 \mathrm{~K}$ эта $I$ уже не изменяется вплоть до $2075 \mathrm{~K}$, что подтверждает принадлежность пика карбиду $\mathrm{W}_{2} \mathrm{C}$. C начала прогрева растет и $I$ пика при $12.1 \mathrm{~V}$, но плавно уменьшая $E$ он при $700 \mathrm{~K}$ смещается к $10.85 \mathrm{~V}$. Однако при $650 \mathrm{~K}$ вновь появляется ступенька при $12.05 \mathrm{~V}$, хорошо проявляющаяся при $825 \mathrm{~K}$. И, наконец, при $525 \mathrm{~K}$ вновь после исчезновения при $425 \mathrm{~K}$ появляется пик ДМЭ при $13.3 \mathrm{~V}$. Рост его I прекращается при $650 \mathrm{~K}$. На этом этапе описаны изменения СУРЭ в первой стадии прогрева и начало второй. На рис. 3 (кривая 1) приведен характерный СУРЭ после десорбции $\mathrm{O}$ с верхних уровней. Но на средних до конца этой стадии еще остаются $\mathrm{W}_{2} \mathrm{CO}$ и $\mathrm{W}_{2} \mathrm{C}$. В конце ее почти полностью снимается экранирование и нижнего. Это подтверждается появлением пиков от $\mathrm{Nb}_{2} \mathrm{~W}_{2} \mathrm{O}$ и подложки соответственно при 3.65, 5.64 и $0.8,13.4 \mathrm{~V}$. Плавное смещение пика при $12.1 \mathrm{~V}$ к $10.85 \mathrm{~V}$ с ростом $I$ говорит об одной их природе и правильности интерпретации как отвечающих несвязывающим орбиталям $\mathrm{O}$ в $\mathrm{W}_{2} \mathrm{CO}$ и $\mathrm{W}_{2} \mathrm{O}$.

Начинающееся с прогревом, казалось бы, аномальное для снятия только лишь экранирования нижнего уровня падение I пика при $3.5 \mathrm{~V}$, причем с аномальным же уменьшением при $525 \mathrm{~K} E$ до $3.05 \mathrm{eV}$, теперь уже точно говорит о его принадлежности новому химическому состоянию на верхнем уровне. Учитывая, что $E$ пика при $4.0 \mathrm{~V}$, напротив, при этом возрастает до $4.3 \mathrm{eV}$, остается приписать их оба субоксиду $\mathrm{W}_{3} \mathrm{O}$, т. е. соответственно его поверхностной и объемной связям $[16,17]$. А повышенное в начале прогрева значение $E$ поверхностной связи в $\mathrm{W}_{3} \mathrm{O}(3.5 \mathrm{eV})$ объясняется наложением пика от такой же связи не полностью экранированного при этом $\mathrm{Nb}_{2} \mathrm{~W}_{2} \mathrm{O}$. К началу второй стадии термодесорбции верхний уровень практически очищается от $\mathrm{O}$ и в вакуум открывается средний. При $625 \mathrm{~K}$ пики при 7.5 и $8.85 \mathrm{~V}$ соответственно от $\mathrm{W}_{2} \mathrm{CO}$ и $\mathrm{W}_{2} \mathrm{C}$ достигают $I_{\max }$. $\mathrm{W}_{3} \mathrm{O}$ на ступеньках верхнего уровня разрушается, а освободившийся $\mathrm{O}$ стекает на те террасы, которые по указанным выше причинам не занимались $\mathrm{W}_{2} \mathrm{CO}$. При этом в СУРЭ появляется новый пик при $3.3 \mathrm{~V}$. $\mathrm{O}$ наличии $\mathrm{O}$ на этих террасах говорит начинающееся при последующем повышении $T_{h}$ падение $I$ этого пика и рост его $E$. Первое отвечает десорбции О с террас, а последнее - началу снятия экранирования нижнего уровня, занятого $\mathrm{Nb}_{2} \mathrm{~W}_{2} \mathrm{O}$. О последнем также говорит появление при $525 \mathrm{~K}$ пика ДМЭ с $E=13.5 \mathrm{eV}$ с последующим ростом $I$ и при $675 \mathrm{~K}$ - пика при $2.7 \mathrm{~V}$ от адсорбата на поверхностных дефектах. При $700 \mathrm{~K}$ десорбируется $\mathrm{O}$ на тех террасах среднего уровня, где не может адсорбироваться СО. В результате исчезает пик при $3.5 \mathrm{~V}$ (рис. 3, кривая 2). При этом пик при $4.25 \mathrm{~V}$, 
отвечающий объемной связи в верхнем субоксиде $\mathrm{W}_{3} \mathrm{O}$, с уменьшением $E$ достигает $3.65 \mathrm{eV}$, теперь уже отвечая поверхностной связи в нижнем $\mathrm{Nb}_{2} \mathrm{~W}_{2} \mathrm{O}$. При этой же $T_{h}$ пик при $12.1 \mathrm{~V}$ плавно достигает $10.85 \mathrm{~V}$, теперь уже отвечая $\mathrm{O}$ на $\mathrm{Nb}_{2} \mathrm{~W}_{2} \mathrm{O}$. Но так как при этой $T_{h}$ еще не полностью десорбируется $\mathrm{CO}$, то с $650 \mathrm{~K}$ несвязывающим орбиталям $\mathrm{O}$ в $\mathrm{W}_{2} \mathrm{CO}$ начинает отвечать новый пик при $12.05 \mathrm{~V}$. Поскольку СО может занимать террасы различных уровней, что не должно отражаться на $E$ внутренней связи С-O, начиная с $650 \mathrm{~K} I$ пика при $7.3 \mathrm{~V}$ от этой связи монотонно уменьшается, достигая $I_{\min }$ при $1325 \mathrm{~K}$, когда по данным ЭОС подложка очищается от CO (рис. 3, кривая 3) [24]. Такой характер изменения I говорит об отсутствии дискретности в наборе уровней рельефа для $\mathrm{CO}$, т.е. большой вероятности его адсорбции на спиральных террасах винтовых дислокаций. С $675 \mathrm{~K}$ и вплоть до $T_{h \text { max }} I$ пика при $8.5 \mathrm{~V}$ от $\mathrm{W}_{2} \mathrm{C}$ не изменяется. Рост же $I$ этого пика в 2 раза в интервале 295-625 К говорит просто о снятии экранирования W при десорбции верхнего О так же, как и об экранировании карбида на верхнем уровне. К концу второй стадии термодесорбции $(\sim 925 \mathrm{~K})$, когда прекращается сильное уменьшение $\varphi$, адсорбат практически покидает и усредненный средний уровень. При этом прекращается и рост $I$ пиков при 5.65 и $10.85 \mathrm{~V}$ соответственно от $\mathrm{Nb}_{2} \mathrm{~W}_{2} \mathrm{O}$ и О на нем. Кроме того, с 725 до $1225 \mathrm{~K}$ не изменяется $I$ другого пика от $\mathrm{Nb}_{2} \mathrm{~W}_{2} \mathrm{O}$ при $3.65 \mathrm{~V}$, а с 750 до $1425 \mathrm{~K}$ - и пика при $0.75 \mathrm{~V}$ от поляризованной на границах островков О или $\mathrm{W}_{2} \mathrm{O}$ подложки. С $975 \mathrm{~K}$ растет I пика ДМЭ при $13.35 \mathrm{~V}$, говоря о начале очистки от O нижнего уровня. При этом $I$ пиков при 7.35 и $15.3 \mathrm{~V}$ падает, а при $2.3 \mathrm{~V}$ растет. Значит, продолжается десорбция $\mathrm{CO}$ и О, причем О покидает еще и объемные дефекты, скорее всего, из верхнего слоя многослойного покрытия. При этой же $T_{h}$ прекращается рост пика от объемных связей $\mathrm{Nb}_{2} \mathrm{~W}_{2} \mathrm{O}$ и от $3.65 \mathrm{~V}$ начинается рост до $3.95 \mathrm{eV}$ при $T_{h \max }$ энергии поверхностной связи. Значит, дополнительный $\mathrm{O}$ на $\mathrm{Nb}_{2} \mathrm{~W}_{2} \mathrm{O}$ уходит с него в поверхностные дефекты, но десорбируется слабо, так как $I$ пика при $2.2 \mathrm{~V}$ от $\mathrm{W}_{2} \mathrm{O}$ начинает падать только после $T_{h}=1975 \mathrm{~K}$. Небольшая очистка $\mathrm{Nb}_{2} \mathrm{~W}_{2} \mathrm{O}$ от верхнего О происходит при 1275-1375 К. При этом после некоторой стабилизации вновь начинают расти $I$ пиков от $\mathrm{Nb}_{2} \mathrm{~W}_{2} \mathrm{O}$. Это может означать, как и на дефектах $\mathrm{W}(110)$, что часть $\mathrm{O}$ на террасах, уже освободившихся от $\mathrm{CO}$, может приводить к образованию дополнительного $\mathrm{Nb}_{2} \mathrm{~W}_{2} \mathrm{O}$ [1-8]. В этом же интервале $T_{h}$ вновь начинается рост $I$ пика при $10.85 \mathrm{~V}$. Поскольку трудно предположить возврат $\mathrm{O}$ на $\mathrm{Nb}_{2} \mathrm{~W}_{2} \mathrm{O}$ нижнего уровня при более высоких $T$, чем $925 \mathrm{~K}$, следует считать, что адсорбция верхнего О в этом случае происходит одновременно на дополнительно образующихся на среднем уровне молекулах $\mathrm{Nb}_{2} \mathrm{~W}_{2} \mathrm{O}$. После $1175 \mathrm{~K}$ начинается рост $I$ пика при $12.5 \mathrm{~V}$, который не прекращается до $T_{\max }$. При более низких $T_{h}$ этот пик, как уже указывалось, может принадлежать несвязывающим орбиталям О как в $\mathrm{W}_{2} \mathrm{O}$, так и в $\mathrm{W}_{2} \mathrm{CO}$. Хотя при $T_{h}=1325-1375 \mathrm{~K} \mathrm{CO}$ десорбируется (I пика при $7.5 \mathrm{~V}$ падает до ступеньки), но
$I$ пика с $E_{b}=2.7 \mathrm{eV}$ растет с уменьшением $E$ до $2.1 \mathrm{eV}$. Значит, на освободившиеся от $\mathrm{CO}$ места поступает $\mathrm{O}$ с более нижних уровней. При этом величина его $E_{b} \mathrm{c}$ подложкой, естественно, становится меньше, а $E$ несвязывающих орбиталей, напротив, больше, приближаясь к $E_{b} 2 p$-уровня изолированного атома $\mathrm{O}(13.62 \mathrm{eV})$. Значит, более высокие значения $E(2.9-2.45 \mathrm{eV})$ для пика с $E_{m} \sim 2.5 \mathrm{eV}$ соответствуют связям $\mathrm{W}-\mathrm{C}-\mathrm{W}$ в $\mathrm{W}_{2} \mathrm{CO}$, а более низкие $(2.4-2.05 \mathrm{eV})-$ связям $\mathrm{W}-\mathrm{O}-\mathrm{W}$ в $\mathrm{W}_{2} \mathrm{O}$. Граничной $T_{h}$ между ними является $900 \mathrm{~K}$, когда пик при $12.05 \mathrm{~V}$ превращается из ступеньки в максимум. Когда при $T_{h}>1875 \mathrm{~K}$ на поверхность из объемных дефектов выходят достаточные количества О и С и последний во время остывания образца может частично окисляться до $\mathrm{CO}$, для некоторых участков подложки в СУРЭ могут отдельно наблюдаться сразу два пика при $2.05-2.3 \mathrm{~V}$ и $2.45-2.8 \mathrm{~V}$ (рис. 3, кривая 4). Таким образом, оказывается разрешенной начальная неопределенность в интерпретации пика с $E_{m} \sim 2.5 \mathrm{eV}$. Последний этап термодесорбции $\mathrm{O}$, начинающийся после полной десорбции СО при $1375 \mathrm{~K}$, отвечает частичному распаду дополнительно образовавшегося $\mathrm{Nb}_{2} \mathrm{~W}_{2} \mathrm{O}$. При этом падает $I$ его пиков при 3.75 и $5.75 \mathrm{~V}$. Но одновременное увеличение их $E$ соответственно до 3.8 и $5.8 \mathrm{eV}$ после $T_{h}=1575 \mathrm{~K}$ и до 4.0 и $5.9 \mathrm{eV}$ после $1975 \mathrm{~K}$ указывает на то, что субоксид разрушается вначале на участках меньшей протяженности и остается на гладких участках большей площади. О последнем говорит и величина $\Delta E=0.65 \mathrm{eV}$ в ПСИВЭ для первого пика. Объяснение же меньшему $\Delta E$ для второго дано выше. При $T=1375 \mathrm{~K}$ происходит уже заметная десорбция О и из неупорядоченных дефектов нижнего уровня. Об этом говорит падение $I$ пика при $10.85 \mathrm{~V}$. Кроме того, начинается интенсивный выход О и из объемных дефектов, о чем говорит падение $I$ пика при $15.4 \mathrm{~V}$. Прослеживаются даже два типа таких дефектов, так как начиная с $1375 \mathrm{~K}$ уже наблюдается дублет с $E=15.0$ и $15.5 \mathrm{eV}, E$ которого растут с $T_{h}$ и после $1525 \mathrm{~K}$ достигают соответственно 15.3 и $15.8 \mathrm{eV}$. После $1875 \mathrm{~K}$ эти пики приходят к $I_{\min }$. Этим подтверждается описанная выше природа понижения $\varphi$ при охлаждении образца после прогрева до высоких $T_{h}$. Рост пиков при 2.2 и $15.4 \mathrm{~V}$ продолжается только до $1575 \mathrm{~K}$. Это означает, что десорбируется не весь О, выходящий из объемных дефектов, а часть его остается на террасах, в том числе и в желобах. Об этом говорит и величина $\Delta E=0.65 \mathrm{eV}$ для пика при $2.2 \mathrm{~V}$ в ПСИВЭ. Но после $T_{h}=1975 \mathrm{~K} \mathrm{I}$ и этих пиков начинает падать. При $1475 \mathrm{~K}$ начинаются падение $I$ пика при $0.65 \mathrm{~V}$, а с $1575 \mathrm{~K}$ - рост интенсивности минимума при $1.35 \mathrm{~V}$, причем после $1975 \mathrm{~K}$ она резко возрастает. Таким образом, рассматривая процессы относительно начального состояния подложки, можно сделать вывод об эффективном удалении с нее $\mathrm{O}$ только начиная с последней $T_{h}$. По данным НЭСУРЭ, более чем в сто раз чувствительной к О, чем ЭОС $[16,17]$, полная очистка от О только совершенных участков на $\mathrm{W}(110)$ происходит при $1100 \mathrm{~K}$, но даже и при этой температуре дефектные участки все еще содержат О [10-15]. Данные 
НЭПСИВЭ, полученные после $T_{h}=2075 \mathrm{~K}$, подтверждают представления, основанные на данных НЭСУРЭ, о состоянии $\mathrm{O}$ на последней стадии десорбции. Так, для пика при $0.6 \mathrm{~V} \Delta E=0$, что говорит об аморфности отвечающего ему О. Пики же при 2.15 и $3.7 \mathrm{~V}$ дают $\Delta E=0.65 \mathrm{eV}$, отвечая наличию упорядоченного О на достаточно протяженных участках. Для первого пика это могут быть и желоба линейных террас, образование которых вдоль образующей облегчается цилиндрической формой поверхности образца [10-15,24]. Но в $I$ пика при $5.8 \mathrm{~V} \mathrm{c} \Delta E=0.3 \mathrm{eV}$ большой вклад уже вносит $\mathrm{O}$ в объемных дефектах. Пик же при $10.85 \mathrm{~V}$ имеет $\Delta E=0.25 \mathrm{eV}$, что говорит о присутствии $\mathrm{O}$ не только на террасах, но и в неупорядоченных дефектах. Пики же при 7.25 и $8.8 \mathrm{~V}$ дают $\Delta E=0.1 \mathrm{eV}$, означающий, что на этой стадии остались в основном малые островки или цепочки из молекул $\mathrm{W}_{2} \mathrm{CO}$ и $\mathrm{W}_{2} \mathrm{C}$. Интересно, что для пленки с $\Delta \varphi_{\max } \Delta E$ этих пиков достигает $0.3 \mathrm{eV}$, что говорит о присутствии этих молекул соответственно на основаниях и в желобах террас, подтверждая тем самым перенос на них С кислородом. А $\Delta E$ пика при $11.7 \mathrm{~V}$, несмотря на уменьшение из-за $\mathrm{O} E \mathrm{c} 12.5 \mathrm{eV}$, достигающий при $\Delta \varphi_{\max } 0.55 \mathrm{eV}$, говорит о том, что на террасах, кроме $\mathrm{W}_{2} \mathrm{CO}$ на основаниях, может быть и $\mathrm{W}_{2} \mathrm{O}$, скорее всего в линейных желобах. В этих же условиях для пика при $3.55 \mathrm{~V} \Delta E=0$, подтверждая наличие малых островков $\mathrm{W}_{3} \mathrm{O}$ на плоскостях самых верхних ступенек, к тому же еще разупорядоченных в азимутальных направлениях, т.е. не имеющих дальнего порядка.

\section{Кислород на нагретой подложке}

При адсорбции О на нагретой подложке при выявлении общих закономерностей для химических состояний, получаемых при $T_{\mathrm{O}}$ в интервале $775-1575 \mathrm{~K}$, установлено следующее. Из новых в СУРЭ может появляться только пик при $2.6-3.0 \mathrm{~V}$, если его не было в начальном спектре. Пик при $12.05-12.5 \mathrm{~V}$ при $T_{\mathrm{O}}=775-1125 \mathrm{~K}$ и $T_{\mathrm{O}}>1425 \mathrm{~K}$ исчезает, а при других $T_{\mathrm{O}}$ его $I$ падает в $3.5-4.5$ раз. Уменьшения $E$ пиков не наблюдается. Ha $0.1-0.4 \mathrm{eV}$ может возрастать $E$ пиков при $0.35-0.6 \mathrm{~V}$, отвечающего им минимума при $0.9-1.3 \mathrm{~V}$, пиков при $3.7-4.05,5.65-5.95$ и $10.7-10.9 \mathrm{~V}$. При этом их $E_{\max }$ может соответственно достигать $0.95,1.6,4.35,5.95$ и $10.95 \mathrm{eV}\left(11.05 \mathrm{eV}\right.$ только для $\left.T_{\mathrm{O}}=775 \mathrm{~K}\right)$.

При сравнении $I$ установлено, что ее рост всегда происходит для пиков при $0.6,2.7,7.3,8.8$ и $15.4 \mathrm{~V}$ и в основном для пиков при 2.2 и $10.8 \mathrm{~V}$. Для всех остальных, в том числе и для минимума при $1.2 \mathrm{~V}$, она падает. При анализе только указанных закономерностей уже можно сделать ряд важных выводов. Главным из них является отсутствие окисления $\mathrm{W}$ покрытия при $P_{\mathrm{O}}<5 \cdot 10^{-5} \mathrm{~Pa}$, которому соответствует $P_{\text {res }} \sim 10^{-3} \mathrm{~Pa}$. Это подтверждается отсутствием роста $I$ пиков в интервале $3.5-6.5 \mathrm{~V}$, отвечающих $E_{b}$ в оксидах. Более того, $I$ пиков при 4.0 и $\sim 6.8 \mathrm{~V}$ от субоксидов, которые при окислении должны бы переходить в оксиды [1-8,10-15], на самом деле падает для первого в 1.5 раза, а для второго в $\sim 2.5$ раза. Это говорит лишь об экранировании субоксида $\mathrm{Nb}_{2} \mathrm{~W}_{2} \mathrm{O}$ дополнительным Q. Более убедительно об отсутствии окисления и преимущественной адсорбции О в объемных дефектах говорит рост $I$ пика при $15.4 \mathrm{~V}$. Например, для $T_{\mathrm{O}}=775 \mathrm{~K}$ она возрастает в 42 раза, для $T_{\mathrm{O}}=925 \mathrm{~K}-$ в 22 раза, для $T_{\mathrm{O}}=1025-1175 \mathrm{~K}-$ в 10 раз, а для $T_{\mathrm{O}}=1575 \mathrm{~K}$ эта $I=I_{0}$. Если рассмотреть те же явления с точки зрения изменения состояния вначале чистых участков подложки, то описанный процесс подтверждается ростом $I$ пика при $0.6 \mathrm{~V}$ в $8-2$ раз соответственно при $T_{\mathrm{O}}=775-1325 \mathrm{~K}$, уменьшением минимума, соответствующего возбуждению тех же электронов, в 7-2 раз, т. е. общее изменение $I$ составляет $15-4$ раз.

Изменение $I$ пика при $2.2 \mathrm{~V}$ от связи $\mathrm{W}-\mathrm{O}-\mathrm{W}$ в $\mathrm{W}_{2} \mathrm{O}$ слабо коррелирует с изменением $I$ пика при $10.8 \mathrm{~V}$, вклад в который могут вносить несвязывающие орбитали как $\mathrm{W}_{2} \mathrm{O}$, так и $\mathrm{O}$ в верхнем слое на $\mathrm{Nb}_{2} \mathrm{~W}_{2} \mathrm{O}$. При $T_{\mathrm{O}} \leq 1225 \mathrm{~K}$ его $I$ падает в 1.5 раза, а после $T_{\mathrm{O}}=1275 \mathrm{~K}$ растет во столько же раз. Значит, больший вклад в $I$ этого пика вносит $\mathrm{O}$ на $\mathrm{Nb}_{2} \mathrm{~W}_{2} \mathrm{O}$, поскольку на сильно пересеченном рельефе при относительно низких $T_{\mathrm{O}}$ он на нижнем уровне может экранироваться. С другой стороны, малое уменьшение $I$ этого пика говорит о том, что в отличие от $T_{\mathrm{O}}=295 \mathrm{~K}$ при $T_{\mathrm{O}}=775-1225 \mathrm{~K}$ адсорбция О на верхних уровнях незначительна. Выше показано, что во время адсорбции О при $295 \mathrm{~K}$ на подложке увеличивается содержание $\mathrm{CO}$ и $\mathrm{W}_{2} \mathrm{C}$ и указаны причины этого явления. Поэтому при повышении $T_{\mathrm{O}}$ до определенного предела возможно некоторое усиление этого эффекта. Действительно, при адсорбции О на нагретом образце в СУРЭ сильно увеличивается $I$ пика при $7.3 \mathrm{~V}$ от $\mathrm{CO}$ и в 2 раза менее сильно при $8.8 \mathrm{~V}$ от $\mathrm{W}_{2} \mathrm{C}$. Значит, в первом приближении при окислении $\mathrm{W}_{2} \mathrm{C} \mathrm{CO}$ образуется в два раза больше, чем при переносе $\mathrm{C}$ выделяется вторичного $\mathrm{W}_{2} \mathrm{C}$. Одновременно растет и I пика при $2.7 \mathrm{~V}$ от связи $\mathrm{W}-\mathrm{C}-\mathrm{W}$ в $\mathrm{W}_{2} \mathrm{CO}$, a если его не было в начальном спектре, то при адсорбции на нагретом образце он появляется, причем совместный рост его $I$ и $E$ до $3.3 \mathrm{eV}$ говорит об увеличении площади, занимаемой пленкой в упорядоченном состоянии. Рост $I$ пика при $8.8 \mathrm{~V}$ в $1.5-3$ раза при $T_{\mathrm{O}}>775 \mathrm{~K}$ говорит об увеличении на подложке выделения $\mathrm{W}_{2} \mathrm{C}$, причем при более низких $T_{\mathrm{O}}$ оно выше. Причины этого описаны выше. На монокристаллах же после окисления карбидов этот пик не наблюдается. При $T_{\mathrm{O}}=775-875 \mathrm{~K}$ для пика при $2.2 \mathrm{~V}$ наблюдается значительно больший рост $I$, чем для $2.7 \mathrm{~V}$, хотя в СУРЭ начального состояния они приблизительно одинаковы (рис. 3, кривая 4), т.е. при этой $T_{\mathrm{O}}$ адсорбция $\mathrm{O}$ преобладает над окислением $\mathrm{W}_{2} \mathrm{C}$. И лишь начиная с $T_{\mathrm{O}}=925 \mathrm{~K}$ картина существенно меняется. При этом пики при $2.45 \mathrm{~V}$ становятся а 2 раза меньше, а при $3.3 \mathrm{~V}-$ в 3 раза больше начальных. Именно с этой $T_{\mathrm{O}}$ прекращается рост $I$ пика при $8.8 \mathrm{~V}$, т. е. эта $T_{\mathrm{O}}$ является оптимальной для окисления карбида. Но при этом еще не происходит заметной десорбции $\mathrm{CO}$, которая интенсивна лишь при $T_{h}=1325-1375 \mathrm{~K}$ 
(рис. 3, кривая 3) [18], т.е. для эффективной очистки от С в О $T$ должна поддерживаться внутри интервала 925-1375 K. Для данного материала она составляла $1100 \pm 25 \mathrm{~K}$ [18]. Однако из-за поликристалличности и, как следствие, большого числа объемных дефектов, постоянная диффузия из них к поверхности С приводит в отличие от монокристаллов [1-8] к невозможности полной очистки подложки от С. Поэтому при получении таких материалов следует повышать требования к технологической и вакуумной гигиене [24].

\section{Заключение}

Таким образом, становится очевидным, что при адсорбции О и $\mathrm{CO}$ на исследуемой поверхности для гладких участков проявляется по крайней мере трехуровневый потенциальный рельеф, который для образных представлений в первом приближении можно считать и геометрическим. С учетом этих трех уровней и описываются в дальнейшем процессы адсорбции, термодесорбции, миграции адсорбатов вдоль подложки и их переходов с уровня на уровень, в том числе и в дефектах или из дефектов различной природы во время прогрева подложки

Даже при высокотемпературной адсорбции О в довольно широком интервале $T_{\mathrm{O}}$, когда следовало бы ожидать окисления подложки, коренного изменения ее начального состояния не происходит. Т.е. W покрытие на сплаве $\mathrm{Nb}-1 \% \mathrm{Zr}$ становится жаростойким. Основной причиной этого являются диффузия $\mathrm{Nb}$ сквозь $\mathrm{W}$ с сегрегацией первого на поверхности в концентрации, не приводящей к металлизации. Присутствие же в материале не удаляемого при высоких $T$ O так же, как и достаточно большие концентрации дополнительного О, приводит только к образованию на базовой поверхности подложки высокотермоустойчивого, как и все субоксиды переходных Ме, интерметаллического субоксида $\mathrm{Nb}_{2} \mathrm{~W}_{2} \mathrm{O}$. Его объемная кристаллическая решетка препятствует окислению оставшегося непрореагировавшим $\mathrm{W}$, который при окислении также должен проходить стадию образования субоксида $\mathrm{W}_{3} \mathrm{O}$ с объемной решеткой. Сохранение последнего для дальнейшего окисления совершенно необходимо [1-8]. С другой стороны, образование субоксида с максимально насыщенными химическими связями $\mathrm{O}-\mathrm{Me}$ между двумя слоями атомов подложки предотвращает дальнейшую сегрегацию на ней $\mathrm{Nb}$, тем самым поддерживая стабильность ее химического состояния. По аналогии с [10-15] можно полагать, что и $\mathrm{Nb}_{2} \mathrm{~W}_{2} \mathrm{O}$ должен быть устойчив по крайней мере до $\sim 2375 \mathrm{~K}$.

\section{Конфликт интересов}

Авторы заявляют, что у них нет конфликта интересов как по этой статье, так и по части самоцитированной литературы.

\section{Список литературы}

[1] Зыков Б.М., Цхакая В.К. // ЖТФ. 1979. Т. 49. Вып. 8. C. $1700-1707$.

[2] Зыков Б.М., Цхакая В.К. // ЖТФ. 1980. Т. 50. Вып. 8. C. $1771-1780$

[3] Зыков Б.М., Нардая Ю.И. // ЖТФ. 1995. Т. 65. Вып. 4. C. $150-166$.

[4] Зыков Б.М., Сабельников А.М., Цхакая В.К., Чилингарашвили Р.С., Ярыгин В.И. // Поверхность. 1983. № 4. C. $65-74$.

[5] Зыков Б.М., Сабельников А.М., Цхакая В.К. // Поверхность. 1986. № 12. С. 21-29.

[6] Зыков Б.М., Сабельников А.М. // Поверхность. 1988. № 10. C. $61-70$.

[7] Зыков Б.М., Сабельников А.М., Цхакая В.К. // Поверхность. 1990. № 6. С. 48-55.

[8] Зыков Б.М., Сабельников А.М. // Поверхность. 1990. № 9. C. $22-29$.

[9] Моррисон С. Химическая физика поверхности твердого тела. М.: Мир, 1980. 488 с.

[10] Зыков Б.М., Сабельников А.М, Цхакая В.К. // Поверхность. 1983. № 9. С. 64-71.

[11] Зыков Б.М., Сабельников А.М., Цхакая В.К. // Поверхность. 1987. № 9. С. 76-83.

[12] Зыков Б.М., Зыкова В.Н., Лебедев В.Н., Удовиченко Ю.К. // Поверхность. 2003. № 7. С. 88-112.

[13] Зыков Б.М., Сабельников А.М. // Высокочистые вещества. 1990. № 5. C. 123-129.

[14] Зыков Б.М., Кобяков В.П., Нардая Ю.И. // Высокочистые вещества. 1991. № 1. С. 71-80.

[15] Зыков Б.М., Нардая Ю.И., Сабельников А.М. // Высокочистые вещества. 1991. № 4. С. 107-115.

[16] Зыков Б.М., Нардая Ю.И.,Сабельников А.М. // Поверхность. 2003. № 8. С. 61-73.

[17] Зыков Б.М., Нардая Ю.И., Сабельников А.М. // РХЖ. 2003. T. 47. № 2. С. 70-80.

[18] Бравареи А.В., Зыков Б.М., Зыкова В.Н., Лебедев В.Н., Удовиченко Ю.К. // ЖТФ. 2002. Т. 72. Вып. 9. С. 112-118.

[19] Зыков Б.М., Цхакая В.К., Ярыгин В.К. Открытия и изобретения. 1983. № 47. С. 236. А.С. № 1062803.

[20] Зыков Б.М., Сабельников А.М. // ПТЭ. 1991. № 1. C. 219-221.

[21] Блейкмор Джс. Физика твердого тела М.: Мир, 1988. $608 \mathrm{c}$.

[22] Крылов О.Ф., Киселев В.Ф. Адсорбция и катализ на переходных металлах и их оксидах. М.: Химия, 1981. $288 \mathrm{c}$.

[23] Семенов И.Н., Овчинников К.В. „Неожиданные“ неорганические соединения. Л.: Химия, 1972. 105 с.

[24] Зыков Б.М., Зыкова В.Н., Лебедев В.Н., Удовиченко Ю.К. // ЖТФ. 2002. Т. 72. Вып. 6. С. 90-100. 